Bull. Korean Math. Soc. 50 (2013), No. 2, pp. 667-674

http://dx.doi.org/10.4134/BKMS.2013.50.2.667

\title{
COMPATIBILITY IN CERTAIN QUASIGROUP HOMOGENEOUS SPACE
}

\author{
Bokhee Im And Ji-Young Ryu
}

\begin{abstract}
Considering a special double-cover $Q$ of the symmetric group of degree 3, we show that a proper non-regular approximate symmetry occurs from its quasigroup homogeneous space. The weak compatibility of any two elements of $Q$ is completely characterized in any such quasigroup homogeneous space of degree 4 .
\end{abstract}

\section{Preliminaries and introduction}

A quasigroup is defined as a set $Q$ equipped with a multiplication, not necessarily associative, such that in the equation

$$
x \cdot y=z,
$$

knowledge of any two of the elements $x, y, z$ of $Q$ specifies the third uniquely. In particular, the solution for $x$ in terms of $y$ and $z$ is written as $z / y$. The body of the multiplication table of a finite quasigroup is a Latin square, while each Latin square may be bordered to give the multiplication table of a quasigroup structure on its set of entries. Nonempty associative quasigroups are groups. A large part of the study of quasigroups aims to extend various aspects of group theory to the more general context of quasigroups [6].

Groups arise from symmetry, understood through transitive group actions. Such actions may be defined equally well for quasigroups, where they lead to the concept of approximate symmetry. Applications in biology and other fields are now raising the problem of developing a rigorous theory of approximate symmetry [5].

Definition $1.1([6])$. Let $P$ be a subquasigroup of a finite, nonempty quasigroup $Q$.

Received January 6, 2012.

2010 Mathematics Subject Classification. Primary 20N05; Secondary 05C69, 20B99.

Key words and phrases. quasigroup, Latin square, action matrix, quasigroup action, homogeneous space, approximate symmetry, intercalate, compatibility.

This research was supported by Basic Science Research Program through the National Research Foundation of Korea(NRF) funded by the Ministry of Education, Science and Technology(2011-0023226). 
(1) The relative left multiplication group $\operatorname{LMlt}_{Q} P$ of $P$ in $Q$ is the subgroup of the symmetric group $\operatorname{Sym} Q$ on the set $Q$ which is generated by all the left multiplications

$$
L(p): Q \rightarrow Q ; x \mapsto p x
$$

for elements $p$ of $P$.

(2) The homogeneous space $P \backslash Q$ is defined as the set of all orbits of $\operatorname{LMlt}_{Q} P$ on $Q$. The cardinality $|P \backslash Q|$ is called the degree $d$ of the homogeneous space $P \backslash Q$. For each element $q$ of $Q$, the right multiplication by $q$ is the permutation

$$
R(q): Q \rightarrow Q ; x \mapsto x q
$$

of $Q$. The action matrix $R_{P \backslash Q}(q)$ of $q$ on $P \backslash Q$ is the $d \times d$ row-stochastic matrix with entry

$$
\left[R_{P \backslash Q}(q)\right]_{X Y}=\frac{|X R(q) \cap Y|}{|X|}
$$

in the row labeled by the $\operatorname{LMlt}_{Q} P$-orbit $X$ and column labeled by the $\operatorname{LMlt}_{Q} P$ orbit $Y$. The homogeneous space $P \backslash Q$ is understood as the set of all orbits of $L_{M l t} P$ on $Q$ together with the action map $q \mapsto R_{P \backslash Q}(q)$.

If $P$ is a subgroup of a finite group $Q$, then the homogeneous space $P \backslash Q$ is the set of right cosets of $P$, and the action matrices become permutation matrices. The quasigroup action may be interpreted as a proper (non-exact) approximate symmetry if at least one action matrix is not a permutation matrix. A proper approximate symmetry has been defined as an exact symmetry holding at least one level of a hierarchial system in $[5,6]$.

We restrict ourselves to the following special case in this paper;

(AS1) a hierarchy with just two levels, so called, macroscopic and microscopic,

(AS2) exact three-fold symmetry only at the macroscopic level as follows:

\begin{tabular}{lc|c|c} 
Macrostates: & $A$ & $B$ & $C$ \\
\hline Microstates: & $a, a^{\prime}$ & $b$ & $c$
\end{tabular}

where $P \backslash Q=\left\{a=P, a^{\prime}, b, c\right\}, \quad A=\left\{a, a^{\prime}\right\}, B=\{b\}, C=\{c\}$. Acknowledging the distinction between $a$ and $a^{\prime}$, however, we will see this symmetry is approximate in the microscopic level.

We consider a quasigroup obtained by permuting entries in the multiplication table of the direct product $S_{3} \times \mathbb{Z}_{2}$ of the symmetric group $S_{3}$ with the 2-element additive group $\mathbb{Z}_{2}$ of integers modulo 2 as in [3], and want to establish a non-regular approximate symmetry from a quasigroup homogeneous space of degree 4 in this paper.

For convenience, let us use the notation $\pi^{\epsilon}$ to denote an element $(\pi, \epsilon)$ of $S_{3} \times \mathbb{Z}_{2}$. Six elements of $S_{3}$ are denoted as three rotations $\rho_{0}=(0), \rho_{1}=(021)$, 
$\rho_{2}=(012)$, and three reflections $\sigma_{0}=(12), \sigma_{1}=(02), \sigma_{2}=(01) . \mathbb{Z}_{2}=\{0,1\}$ as in [3]. Consider a relation $\lambda \cdot \mu=\nu$ in $S_{3}$. The corresponding fragment

$$
\left[\begin{array}{ll}
\nu^{0} & \nu^{1} \\
\nu^{1} & \nu^{0}
\end{array}\right]
$$

of the body of the multiplication table of $S_{3} \times \mathbb{Z}_{2}$, indexed by the respective rows labeled $\lambda^{0}, \lambda^{1}$ and columns labeled $\mu^{0}, \mu^{1}$, is known as the intercalate corresponding to the source $\lambda$ and sink $\mu$.

The body of the multiplication table of the group $S_{3} \times \mathbb{Z}_{2}$ is a Latin square. It remains a Latin square if the intercalate (1.1) is changed to

$$
\left[\begin{array}{ll}
\nu^{1} & \nu^{0} \\
\nu^{0} & \nu^{1}
\end{array}\right]
$$

Altogether, $2^{36}$ quasigroup structures on the set $S_{3} \times \mathbb{Z}_{2}$ are obtained by making such intercalate changes in the body of the multiplication table of the group $S_{3} \times \mathbb{Z}_{2}$. Each such quasigroup is specified uniquely by a directed graph $\Gamma$ (in which loops are allowed) on the vertex set $S_{3}$ : The intercalate corresponding to the source $\lambda$ and sink $\mu$ is changed precisely when there is a directed edge in $\Gamma$ from the source $\lambda$ to the sink $\mu$. Write $Q(\Gamma)$ for the quasigroup specified in this way by a directed graph $\Gamma$. Let $M(\Gamma)=\left[\gamma_{\lambda \mu}\right]$ be the adjacency matrix of $\Gamma$, interpreted as a matrix over the field $\mathbb{Z}_{2}$. Then for $\lambda, \mu$ in $S_{3}$ and $l, m$ in $\mathbb{Z}_{2}$, the equation

$$
\lambda^{l} \cdot \mu^{m}=\nu^{l+m+\gamma_{\lambda \mu}}
$$

specifies the product of $\lambda^{l}$ and $\mu^{m}$ in $Q(\Gamma)$, given the product relation $\lambda \cdot \mu=\nu$ in $S_{3}$. Note that the projection

$$
\theta: Q(\Gamma) \rightarrow S_{3} ; \lambda^{l} \mapsto \lambda
$$

is a quasigroup homomorphism.

Lemmas 2.1 and 2.2 determine which $\Gamma$ yields our homogeneous space $P \backslash Q$ of degree 4. In Theorem 2.3 we see that the action matrices of $Q:=Q(\Gamma)$ are independent of the choices of a directed graph $\Gamma$ and all action matrices of our homogeneous space $P \backslash Q$ are listed completely. Then we completely characterize the weak compatibility of any two elements of $Q$ in Theorem 3.3. However, the strong compatibility of $Q$ still remains to be solved.

As long as strong compatibility of any two elements of $Q$ is characterized in quasigroup homogeneous space of degree 4 , based on that result, we expect to characterize the compatibility completely for the case of degree 6 without much trouble, in which case the homogeneous space is uniform. And such graph-theoretical characterization of compatibility will be used to study sharp tnansitivity in a quasigroup. For further details, see [3]. 


\section{Action matrices}

We follow notations and terminologies as described in the previous section. Let us arrange a quasigroup $Q=S_{3} \times \mathbb{Z}_{2}$ as an ordered set

$$
\left\{\rho_{0}^{0}, \rho_{0}^{1}, \sigma_{0}^{0}, \sigma_{0}^{1}, \rho_{1}^{0}, \rho_{1}^{1}, \sigma_{1}^{0}, \sigma_{1}^{1}, \rho_{2}^{0}, \rho_{2}^{1}, \sigma_{2}^{0}, \sigma_{2}^{1}\right\}
$$

according to the ordered set $S_{3}=\left\{\rho_{0}, \sigma_{0}, \rho_{1}, \sigma_{1}, \rho_{2}, \sigma_{2}\right\}=\{1,2,3,4,5,6\}$ so that we have the exact symmetry in the macroscopic level of our hierarchial system throughout this paper.

Lemma 2.1. Let $Q:=Q(\Gamma)$ be a quasigroup specified by a directed graph $\Gamma$ and let $P$ be a subquasigroup of $Q$. Suppose the homogeneous space $P \backslash Q$ is of degree 4 . Then we have

(1) $P$ must be of order 2 and contain either $\rho_{0}^{0}$ or $\rho_{0}^{1}$ but not both,

(2) If $\rho_{0}^{\varepsilon} \in P$, then $\gamma_{\rho_{0} \rho_{0}}=\varepsilon$, where $\varepsilon=\{0,1\}$.

Proof. (1) We must exclude the case of $P$ being $\left\{\rho_{0}^{0}, \rho_{0}^{1}\right\}$ which implies that $P \backslash Q$ is isomorphic to the group $S_{3}$.

(2) If $\rho_{0}^{\varepsilon} \in P$, then by definition of $\gamma_{\lambda \mu}$ in (1.2), we have $\rho_{0}^{\varepsilon} \rho_{0}^{\varepsilon}=\rho_{0}^{\gamma_{1,1}}$ which must be in $P$, hence we have $\gamma_{1,1}=\varepsilon$.

By the above lemma, we should have $P$ as only one of the following four subsets $\left\{\rho_{0}^{0}, \sigma_{i}^{0}\right\},\left\{\rho_{0}^{0}, \sigma_{i}^{1}\right\},\left\{\rho_{0}^{1}, \sigma_{i}^{0}\right\},\left\{\rho_{0}^{1}, \sigma_{i}^{1}\right\}$ for each $i=0,1,2$. Without loss of generality we assume $i=0$ in this paper. Our homogeneous space $P \backslash Q$ only depends on the first two rows of the adjacency matrix of $\Gamma$ by the definition.

Lemma 2.2. Let $Q=Q(\Gamma)$ be a quasigroup and let $P \backslash Q$ be the homogeneous space of degree 4. Then we can interpret the homogeneous space $P \backslash Q$ to have three states $A:=\left\{\rho_{0}^{0}, \rho_{0}^{1}, \sigma_{0}^{0}, \sigma_{0}^{1}\right\}, B:=\left\{\rho_{1}^{0}, \rho_{1}^{1}, \sigma_{1}^{0}, \sigma_{1}^{1}\right\}$, and $C:=\left\{\rho_{2}^{0}, \rho_{2}^{1}, \sigma_{2}^{0}, \sigma_{2}^{1}\right\}$ in the macroscopic level, and four states $P, A-P, B, C$ in the microscopic level. Moreover, we need the following restrictions only in the first two rows of the adjacency matrix of $\Gamma$ in each case;

(1) If $\rho_{0}^{0} \in P$, then the minor matrix $\left[\begin{array}{ll}\gamma_{1,1} & \gamma_{1,2} \\ \gamma_{2,1} & \gamma_{2,2}\end{array}\right]$ of the adjacency matrix of $\Gamma$ is $\left[\begin{array}{ll}0 & 0 \\ 0 & 0\end{array}\right]$ and the minor matrix $\left[\begin{array}{ll}\gamma_{1, s} & \gamma_{1, s+1} \\ \gamma_{2, s} & \gamma_{2}, s+1\end{array}\right]$ of the adjacency matrix of $\Gamma$ is neither $\left[\begin{array}{ll}0 & 0 \\ 0 & 0\end{array}\right]$ nor $\left[\begin{array}{ll}0 & 0 \\ 1 & 1\end{array}\right]$ for $s=3,5$.

(2) If $\rho_{0}^{1} \in P$, then the minor matrix $\left[\begin{array}{ll}\gamma_{1,1} & \gamma_{1,2} \\ \gamma_{2,1} & \gamma_{2,2}\end{array}\right]$ of the adjacency matrix of $\Gamma$ is $\left[\begin{array}{ll}1 & 1 \\ 1 & 1\end{array}\right]$ and the minor matrix $\left[\begin{array}{ll}\gamma_{1, s} & \gamma_{1, s+1} \\ \gamma_{2, s} & \gamma_{2}, s+1\end{array}\right]$ of the adjacency matrix of $\Gamma$ is neither $\left[\begin{array}{ll}1 & 1 \\ 1 & 1\end{array}\right]$ nor $\left[\begin{array}{ll}1 & 1 \\ 0 & 0\end{array}\right]$ for $s=3,5$.

Proof. (1) By lemma 2.1, if $\rho_{0}^{0} \in P$, then $\gamma_{1,1}=0$. And since $|P|=2$, macrostate $A$ consists of two orbits $P$ and $A-P$, where $P$ is either $\left\{\rho_{0}^{0}, \sigma_{0}^{0}\right\}$ or $\left\{\rho_{0}^{0}, \sigma_{0}^{1}\right\}$. So we should have $\sigma_{0}^{\varepsilon} \rho_{0}^{0}=\sigma_{0}^{\varepsilon}=\sigma_{0}^{\varepsilon+\gamma_{2,1}}$, hence we have $\gamma_{2,1}=0$. Similary, $\gamma_{1,2}=\gamma_{2,2}=0$. There are $2^{4}$ possibilities to be checked. And suppose $\{0,1\}=\{\varepsilon, \tau\}$. If the minor matrix $\left[\begin{array}{ll}\gamma_{1, s} & \gamma_{1, s+1} \\ \gamma_{2, s} & \gamma_{2, s+1}\end{array}\right]=\left[\begin{array}{ll}0 & 0 \\ 0 & 0\end{array}\right]$, the macrostate $B$ divides into two orbits $\left\{\rho_{1}^{0}, \sigma_{1}^{\varepsilon}\right\},\left\{\rho_{1}^{1}, \sigma_{1}^{\tau}\right\}$ if $\sigma_{0}^{\varepsilon} \in P$. Now let's check for the rest of $2^{4}-1$ choices. If $\gamma_{1, s}=1$, then $\rho_{0}^{0} \rho_{1}^{0}=\rho_{1}^{1}$, hence the orbit of $\rho_{1}^{0}$ becomes $B$. Similarly $B$ is the orbit of $\sigma_{1}^{0}$ for the case when $r_{1, s+1}=1$. Now let's 
consider when $\gamma_{1, s}=\gamma_{1, s+1}=0$. If $\gamma_{2, s}=1 \neq 0=r_{2, s+1}$, then $\sigma_{0}^{\varepsilon} \rho_{1}^{0}=\sigma_{1}^{\tau}$ and $\sigma_{0}^{\varepsilon} \sigma_{1}^{1}=\rho_{1}^{\tau}$, i.e., $\rho_{1}^{0}$ and $\rho_{1}^{1}$ are in the same orbit $B$. Hence this case is allowable. Similarly the case when $\gamma_{2, s}=0 \neq 1=\gamma_{2, s+1}$ is allowable. However we need to exclude the case of $\gamma_{2, s}=\gamma_{2, s+1}=1$, where $B$ consists of two orbits $\left\{\rho_{1}^{0}, \sigma_{1}^{\tau}\right\}$, $\left\{\rho_{1}^{1}, \sigma_{1}^{\varepsilon}\right\}$ if $\sigma_{0}^{\varepsilon} \in P$.

(2) is analogous to the case of (1).

Theorem 2.3. Let $Q=Q(\Gamma)$ be a quasigroup and let the homogeneous space $P \backslash Q$ be an ordered set $\{P, A-P, B, C\}$. Action matrices of $Q(\Gamma)$ are independent of the choices of $P$ and of a directed graph $\Gamma$, and

(1) the action matrices of elements in $A$ are precisely one of the following

$$
\left[\begin{array}{cc}
I & O \\
O & I
\end{array}\right], \quad\left[\begin{array}{cc}
T & O \\
O & I
\end{array}\right], \quad\left[\begin{array}{ll}
I & O \\
O & T
\end{array}\right], \quad\left[\begin{array}{ll}
T & O \\
O & T
\end{array}\right]
$$

(2) the action matrices of elements in $B$ or $C$ are precisely one of the following

$$
\left[\begin{array}{ccc}
O & \mathbf{j} & \mathbf{0}^{\mathbf{t}} \\
\mathbf{0} & 0 & 1 \\
\mathbf{u} & 0 & 0
\end{array}\right],\left[\begin{array}{ccc}
O & \mathbf{0}^{\mathbf{t}} & \mathbf{j} \\
\mathbf{u} & 0 & 0 \\
\mathbf{0} & 1 & 0
\end{array}\right],\left[\begin{array}{ccc}
O & \mathbf{j} & \mathbf{0}^{\mathbf{t}} \\
\mathbf{u} & 0 & 0 \\
\mathbf{0} & 0 & 1
\end{array}\right],\left[\begin{array}{ccc}
O & \mathbf{0}^{\mathbf{t}} & \mathbf{j} \\
\mathbf{0} & 1 & 0 \\
\mathbf{u} & 0 & 0
\end{array}\right]
$$

where

$$
\begin{aligned}
& I=\left[\begin{array}{ll}
1 & 0 \\
0 & 1
\end{array}\right], \quad T=\left[\begin{array}{ll}
0 & 1 \\
1 & 0
\end{array}\right], \quad O=\left[\begin{array}{ll}
0 & 0 \\
0 & 0
\end{array}\right], \\
& \mathbf{0}=\left[\begin{array}{ll}
0 & 0
\end{array}\right], \quad \mathbf{u}=\left[\begin{array}{ll}
\frac{1}{2} & \frac{1}{2}
\end{array}\right], \quad \mathbf{j}=\left[\begin{array}{l}
1 \\
1
\end{array}\right] .
\end{aligned}
$$

Proof. Suppose a subquasigroup $P$ is any of our 4 cases.

(1) If $\rho_{0}^{\varepsilon}$ is in $\mathrm{P}$, then $\left[\begin{array}{lll}\gamma_{1,1} & \gamma_{1,2} \\ \gamma_{2,1} & \gamma_{2,2}\end{array}\right]=\left[\begin{array}{ll}\varepsilon & \varepsilon \\ \varepsilon & \varepsilon\end{array}\right]$ by Lemma 2.2. Hence the identity matrix is the action matrix corresponding to $q=\rho_{0}^{\varepsilon} \in P$. And the second matrix listed in Lemma corresponds to element $q_{2}$ in $A-P$, where $\theta(q)$ is a rotation. For $q \in A$, if $\theta(q)$ is a reflection in $P$, then the corresponding action matrix of $q$ is the third matrix and if $\theta(q)$ is a reflection in $A-P$, then the corresponding action matrix of $q$ is the fourth matrix.

(2) By definition we get the following 4 matrices only.

$$
\begin{aligned}
& R_{P \backslash Q}\left(\rho_{1}^{0}\right)=R_{P \backslash Q}\left(\rho_{1}^{1}\right)=\left[\begin{array}{lll}
O & \mathbf{j} & \mathbf{0}^{\mathbf{t}} \\
\mathbf{0} & 0 & 1 \\
\mathbf{u} & 0 & 0
\end{array}\right], \\
& R_{P \backslash Q}\left(\rho_{1}^{0}\right)=R_{P \backslash Q}\left(\rho_{2}^{1}\right)=\left[\begin{array}{lll}
O & \mathbf{0}^{\mathbf{t}} & \mathbf{j} \\
\mathbf{u} & 0 & 0 \\
\mathbf{0} & 1 & 0
\end{array}\right], \\
& R_{P \backslash Q}\left(\sigma_{1}^{0}\right)=R_{P \backslash Q}\left(\sigma_{1}^{1}\right)=\left[\begin{array}{lll}
O & \mathbf{j} & \mathbf{0}^{\mathbf{t}} \\
\mathbf{u} & 0 & 0 \\
\mathbf{0} & 0 & 1
\end{array}\right],
\end{aligned}
$$




$$
R_{P \backslash Q}\left(\sigma_{2}^{0}\right)=R_{P \backslash Q}\left(\sigma_{2}^{1}\right)=\left[\begin{array}{ccc}
O & \mathbf{0}^{\mathbf{t}} & \mathbf{j} \\
\mathbf{0} & 1 & 0 \\
\mathbf{u} & 0 & 0
\end{array}\right] .
$$

Note that action matrices of $Q(\Gamma)$ are independent of the choices of $\mathrm{P}$ and of a directed graph $\Gamma$.

Note 2.4. Action matrices of two elements in $P($ resp. $A-P$ ) have the same $(1,1)$-entry $I$ (resp. $T$ ). Note that the (1,2)-entry of action matrix of each element in $B$ is identically $\mathbf{j}=\left[\begin{array}{l}1 \\ 1\end{array}\right]$. For the case of $C,(1,3)$-entry is involved.

Remark 2.5. If the distinction between two microstates $P$ and $A-P$ is ignored, then we obtain exact 3 -fold symmetry between three macrostates $A, B$ and $C$. However, acknowledging the distinction between two microstate $P$ and $A-P$, we see this symmetry is only approximate in the microscopic level. For example, let

$$
M=\left[\begin{array}{ccc}
O & \mathbf{j} & \mathbf{0}^{\mathbf{t}} \\
\mathbf{u} & 0 & 0 \\
\mathbf{0} & 0 & 1
\end{array}\right]=\left[\begin{array}{cccc}
0 & 0 & 1 & 0 \\
0 & 0 & 1 & 0 \\
\frac{1}{2} & \frac{1}{2} & 0 & 0 \\
0 & 0 & 0 & 1
\end{array}\right]
$$

be an action matrix. Then applying an action matrix $M$ to the first microstate $P$ gives the third microstate $B$, but an application of $M$ leads the first state $P$ only with probability a half, otherwise gives the second microstate $A-P$.

\section{Compatibility}

Definition $3.1([3])$. Suppose that $P$ is a subquasigroup of a finite, nonempty quasigroup $Q$.

(a) Two distinct elements $q_{1}$ and $q_{2}$ of $Q$ are said to be strongly compatible (in the action on $P \backslash Q$ ) if

$$
R\left(q_{1}\right)^{-1}(Y) \cap R\left(q_{2}\right)^{-1}(Y)=\varnothing
$$

for all points $Y$ of $P \backslash Q$.

(b) Two distinct elements $q_{1}$ and $q_{2}$ of $Q$ are said to be weakly compatible (in the action on $P \backslash Q$ ) if

$$
\left[R_{P \backslash Q}\left(q_{1}\right)\right]_{X Y}+\left[R_{P \backslash Q}\left(q_{2}\right)\right]_{X Y} \leq 1
$$

for all points $X$ and $Y$ of $P \backslash Q$.

Proposition 3.2. Let $P$ be a subgroup of a finite group $Q$. In the action on $P \backslash Q$, the strong compatibility of two elements $q_{1}, q_{2}$ of $Q$ is equivalent to their weak compatibility.

Now we can completely characterize the weak compatibility of any two elements of $Q$ in the following theorem. 
Theorem 3.3. Let $Q:=Q(\Gamma)$ be a quasigroup and let the homogeneous space $P \backslash Q$ be of degree 4 . Let $q_{1}$ and $q_{2} \in Q$. Then

(1) If both $\theta\left(q_{1}\right)$ and $\theta\left(q_{2}\right)$ are rotations or reflections, but not in the same macrostates $A, B$ or $C$, then $q_{1}$ and $q_{2}$ are weakly compatible.

(2) If $\theta\left(q_{1}\right)$ is a rotation and $\theta\left(q_{2}\right)$ a reflection, then $q_{1}$ and $q_{2}$ are weakly compatible only in the following cases;

(i) $q_{1}$ and $q_{2}$ are both in the macrostate $A$ and in the different orbits $P$ and $A-P$,

(ii) $q_{1}$ and $q_{2}$ are in the different macrostates $B, C$.

Proof. By Note 2.4, any two elements in the same orbit are not weakly compatible. (1) Suppose $\theta\left(q_{1}\right)$ and $\theta\left(q_{2}\right)$ are both rotations or reflections but not in the same macrostates $A, B$ or $C$. Then $q_{1}$ and $q_{2}$ are strongly compatible by the group structure of $S_{3}$. So $q_{1}$ and $q_{2}$ are also weakly compatible.

(2) Let $\theta\left(q_{1}\right)$ be a rotation and let $\theta\left(q_{2}\right)$ be a reflection. (i) From the proof of Theorem 2.3, we note that the action matrix of a rotation in $P$ is the identity matrix, that of a rotation in $A-P$ is a $2 \times 2$ block matrix $\left[\begin{array}{ll}T & O \\ O & I\end{array}\right]$, that of a reflection in $P$ is $\left[\begin{array}{ll}I & O \\ O & T\end{array}\right]$, and that of reflection in $A-P$ is $\left[\begin{array}{cc}T & O \\ O & T\end{array}\right]$. So if $q_{1}$ and $q_{2}$ are both in the macrostate $A$ and in the different orbits $P$ and $A-P$, then $q_{1}$ and $q_{2}$ are weakly compatible. However if $q_{1}$ and $q_{2}$ are both in the same orbits $P$ or $A-P$ of $A$, then (1,1)-block breaks the condition for the weak compatibility of $q_{1}$ and $q_{2}$. (ii) Suppose $q_{1}$ and $q_{2}$ are in the different macrostates $B, C$. By the action matrix of $q_{i} \in B, C$ in the proof of Theorem 2.3 and the definition of weak compatibility, we know that the weak compatibility of two elements $q_{1}$ and $q_{2}$ of $Q$. If $q_{1}$ is in $A$ and $q_{2}$ is in the $B$ or $C$, then $q_{1}$ and $q_{2}$ are not weakly compatible. Indeed, $\left[R_{P \backslash Q}\left(q_{1}\right)\right]_{X Y}+\left[R_{P \backslash Q}\left(q_{2}\right)\right]_{X Y}=2>1$ in case of $X=C$, and $Y=B$ or $C$.

Remark 3.4. The strong compatibility of any two elements of $Q$ still remains to be solved. We need to check the strong compatibility only for those cases mentioned in Theorem 3.3 due to Proposition 3.2. But the main trouble is the case $(2$ - ii) of Theorem 3.3. When this case is also solved, then we expect the result will lead to characterization of the compatibility for the case of quasigroup homogeneous space of degree 6 without too much trouble, in which case the homogeneous space is uniform and such graph-theoretical characterization of compatibility will be used to study sharp tnansitivity in a quasigroup.

\section{References}

[1] P. J. Cameron and C. Y. Ku, Intersecting families of permutations, European J. Combin. 24 (2003), no. 7, 881-890.

[2] K. Heinrich and W. D. Wallis, The maximum number of intercalates in a latin square, Combinatorial mathematics, VIII (Geelong, 1980), pp. 221-233, Lecture Notes in Math., 884, Springer, Berlin-New York, 1981.

[3] B. Im, J.-Y. Ryu, and J. D. H. Smith, Sharply transitive sets in quasigroup actions, J. Algebraic Combin. 33 (2011), no. 1, 81-93. 
[4] B. D. McKay and I. M. Wanless, Most Latin squares have many subsquares, J. Combin. Theory Ser. A 86 (1999), no. 2, 322-347.

[5] J. D. H. Smith, Symmetry and entropy: a hierarchical perspective, Symmetry 16 (2005), $37-45$.

[6] _ An Introduction to Quasigroups and Their Representations, Chapman and Hall/CRC, Boca Raton, FL, 2007.

BOKHEE IM

Department of Mathematics

Chonnam National University

GwanguU 500-757, Korea

E-mail address: bim@jnu.ac.kr

JI-Young RYu

Department of Mathematics

Chonnam National University

GWANGJU 500-757, Korea

E-mail address: rjiyoung1004@hanmail.net 